\title{
Theory of terahertz electric oscillations by supercooled superconductors
}

\author{
Todor M. Mishonov* and Mihail T. Mishonov \\ Department of Theoretical Physics, Faculty of Physics, \\ University of Sofia St. Kliment Ohridski, \\ 5 J. Bourchier Blvd., 1164 Sofia, Bulgaria
}

\begin{abstract}
We predict that below $T_{c}$ a regime of negative differential conductivity (NDC) can be reached. The superconductor should be supercooled to $T<T_{c}$ in the normal phase under DC voltage. In such a nonequilibrium situation the NDC of the superconductor is created by the excess conductivity of the fluctuation Cooper pairs. We propose NDC of supercooled superconductors to be used as an active medium for generation of electric oscillations. Such generators can be used in the superconducting electronics as a new type $\mathrm{THz}$ source of radiation. Oscillations can be modulated by the change of the bias voltage, electrostatic doping by a gate electrode when the superconductor is the channel of a field effect transistor, or by light. When small amplitude oscillations are stabilized near the critical temperature $T_{c}$ the generator can be used as a bolometer. The essential for the applications NDC is predicted by the solution of the Boltzmann kinetic equation for the metastable in the normal phase Cooper pairs. Boltzmann equation for fluctuation Cooper pairs is a result of state-of-the-art application of the microscopic theory of superconductivity. Our theoretical conclusions are based on some approximations like time dependent Ginzburg-Landau theory, but nevertheless can reliably predict appearance of NDC. NDC is the main ingredient of the proposed technical applications. The maximal frequency at which superconductors can operate as generators is determined by the critical temperature $\hbar \omega_{\max } \sim k_{B} T_{c}$. For high- $T_{c}$ superconductors this maximal frequency falls well inside the terahertz range. Technical conditions to avoid nucleation of the superconducting phase are briefly discussed. We suggest that nanostructured high- $T_{c}$ superconductors patterned in a single chip can give the best technical performance of the proposed oscillator.

PACS numbers: $74.40+\mathrm{k}, 85.35 . \mathrm{Kt}, 84.30 . \mathrm{Ng}, 74.78 .-\mathrm{w}$
\end{abstract}

\section{INTRODUCTION}

Due to their low ohmic dissipation superconductors find a lot of technical applications. Superconductors can be used in resonators, cables, electromagnets, transformers, electrical engines and generators. As another important technical application we wish to mention the SQIDs based on Josephson effect. However, up to now there is a little progress in the use of superconductors as active elements in electronic circuits such as amplifiers and generators integrated in superconducting electronics. The present work falls in this still uncompleted technical niche. We are suggesting how superconductors, and especially high- $T_{c}$ superconductors, can be used as generators of high frequency electric oscillations including the $\mathrm{THz}$ range. When the superconductor is implemented as a thin layer the work of the generator can be influenced by the electrostatic charge modulation as in a gate transistor, by heat, light or simply by the change of the DC bias voltage. Such a way oscillations can be modulated, the generator can be used as an optoelectronic device or as a bolometer. The electric oscillations are generated by negative differential conductivity (NDC) of a superconductor in non equilibrium condition. The superconductor is supercooled in the normal phase below the critical temperature $T_{c}$ under a constant electric field. The electric field prevents transition of the superconductor to the superconducting phase state. NDC is coming from so called fluctuation conductivity which is strongly expressed for cuprate superconductors having smaller coherence length.

\section{PHYSICAL MODEL}

\section{A. Qualitative consideration and analogies}

First we will describe the idea how the negative differential conductivity (NDC) can be created in a superconductor. Let us consider the physical processes which happen in a supercooled in the normal state superconductor under voltage. The material is in a normal state and in his volume thermally activated Cooper pairs are continuously created. This stochastic process is analogous to the Brownian motion but is related to the wave function of the metastable Cooper pairs, the Ginzburg-Landau order parameter $\Psi(\mathbf{r}, t)$ which for the normal phase is a time and space-vector dependent stochastic function. When the temperature is below the critical one $T<T_{c}$ both the amplitude of the wave function and the number of fluctuation Cooper pairs increase with the time. Such wave amplification is analogous to the lasing process in lasers or to the dynamics of the Bose condensation. This is the precursor of the transition of the superconductor in the superconducting phase which has infinite conductivity; the electric current can flow without an external voltage in the superconducting phase. However, the applied DC electric field prevents fluctuation Cooper pairs 
to condense in a coherent superconducting phase; superconducting phase cannot exist under external voltage. Once born, the electric field accelerates the fluctuation Cooper pairs and their kinetic energy increases. However, the decay rate of the Cooper pairs is energy dependent and increases with the energy. Like on a highway big velocity increases the probability of accidents. Roughly speaking, the life of faster Cooper pairs is shorter and the electric field finally destroys the accelerated Cooper pairs. During their life metastable Cooper pairs carry significant electric current (fluctuation current) comparable and even bigger than the current of normal charge carriers electrons or holes (normal current); the total current is the sum of fluctuation and normal current.

We consider as very instructive to give also popular explanations using the analogy with ideas from two atmospheric phenomena which have no direct link in real meteorology: rains and meteors; as we stressed it will be only analogy. (1) Imagine a very humid atmosphere supercooled below the condensation temperature. Small droplets continuously grow in size and it begins raining. We have different droplets with different sizes and velocities that create the current density of the rain - a total amount of water passing through a square meter per one second. (2) Second phenomenon is related to meteorites falling from the cosmic space; moving with high velocities stones burn in the atmosphere due to the heating by the friction force. Imagine now that Earth acceleration is many orders of magnitude higher (for the real meteorology it is unrealistic) that makes big rain droplets moving with high velocity evaporate by heating from the friction force in bigger extent. This sets limits to the maximal size for falling droplets and the rain could be a continuous process with a current density determined by the acceleration force. The subtle question here is what will happen if acceleration will slightly decrease. The smaller acceleration will lead to a bigger size of the largest droplets and as a consequence the debit of the rain will increase. This is an example of how NDC appears - small decrease in the driving force cause increasing of the current. The analog of the droplets is the square of modulus of the Fourier components of the effective Ginzburg-Landau function $\left|\Psi_{\mathbf{k}}(t)\right|^{2}$. When a superconductor is cooled below the critical temperature $T_{c}$ in the bulk of the superconductor starts a process similar to Bose condensation. Electrons start to condensate in Cooper pairs and many Cooper pairs can have a common momentum $\hbar \mathbf{k}$ - this is the analog of a rain droplet having a velocity proportional to its momentum.

After this analogy and a qualitative description of the main processes we can analyze in detail the appearance of the NDC - the current increasing when the electric field decreases. Such a behavior is opposite to the ohmic conductivity.

When the electric field is smaller, the acceleration is slower, the decay rate of fluctuation Cooper pairs is slower, and as a result the volume density of the fluctuation Cooper pair is higher and the current is higher too.
The acceleration, creating the decay of Cooper pairs, is an analog of the evaporation of droplets by the heating force from our above-mentioned example. So, the electric field accelerates and destroys Cooper pairs and the superconductor cannot move to superconducting state. Thus it is logically that the electric current density $j(E)$ increases when the electric field $E$ decreases, i.e. the differential conductivity of the material is negative

$$
\varsigma_{\text {diff }}(E)=\frac{d j(E)}{d E}
$$

In such a non equilibrium situation supercooled superconductor will have NDC due to the electric field dependence of the density of metastable Cooper pairs.

Here, by way of illustration we will consider another analogy of the creation of electric oscillations by supercooled superconductors. In some sense, the active part of a superconductor with NDC operates as a laser. However, there is a need to pump the energy in the active medium in order to make lasers operating. Now, let us consider how the superconductor is lasing. Below $T_{c}$, the ground state of the material is the superconducting state having the lowest free energy and the normal state (nonsuperconducting state with an Ohmic resistivity) is the "excited state" having higher energy and generating electric oscillations. The energy for these oscillations comes from the constant electric field but in some sense the excited state is reached by cooling. For high- $T_{c}$ superconductors the cooling can be done by liquid nitrogen that is substantially cheaper. The initial demonstrations of the effect of the NDC, however, can be realized at helium temperatures by technologically more convenient conventional superconductors.

Like the current, the total differential conductivity is a sum of its fluctuation and normal parts. The normal part of the differential conductivity $\varsigma_{N}(T)$ weakly depends on the electric field. In the next subsection we will describe the state-of-the-art theory of the differential conductivity of the fluctuation Cooper pairs.

\section{B. Formulas for the differential conductivity}

For a comprehensive contemporary review on the properties of superconductors see the book edited by Benneman and Ketterson which starts with a review article by Larkin and Varlamov on the fluctuation phenomena in superconductors $\stackrel{1}{\underline{1}}$ There can be found detailed explanations of the Bardeen, Cooper and Schrieffer (BCS) theory of superconductivity and also of the time dependent Ginzburg-Landau (TDGL) theory for the order parameter of superconductors. Ref. 2 is another review especially devoted to the Gaussian fluctuation in superconductors. The Boltzmann equation for the fluctuation Cooper pairs was derived ${ }^{3}$ in the framework of TDGL theory. For the case of strong electric fields Boltzmann equation was solved in Ref. [4, and a general formula for the fluctuation current was derived as well; see also the 
references therein. The same result was rederived ${ }^{5}$ directly from the TDGL theory. Our formula for small electric fields below $T_{c}$ is similar to the formula by Go'kov (1970) which was however, directly derived ${ }^{6}$ within the framework of BCS theory; see also the work by Tucker and Halperin ${ }^{7}$ Differentiating the formula for the fluctuation current $\mathrm{t}^{4.5}$

$$
j_{\mathrm{fl}}\left(E_{x}\right)=\frac{e^{2} \tau_{\mathrm{rel}} E_{x}}{16 \hbar\left[\pi^{1 / 2} \xi(0)\right]^{D-2}} \int_{0}^{\infty} \frac{\exp \left(-\epsilon u-g u^{3}\right)}{u^{(D-2) / 2}} d u,
$$

we obtain the formula for the total differential conductivity

$$
\begin{aligned}
\varsigma_{\text {diff }}(E)=\varsigma_{N}(T) & +\frac{e^{2} \tau_{\text {rel }}}{16 \hbar\left[\pi^{1 / 2} \xi(0)\right]^{D-2}} \\
& \times \int_{0}^{\infty} \frac{\exp \left(-\epsilon u-g u^{3}\right)}{u^{(D-2) / 2}}\left(1-2 g u^{3}\right) d u
\end{aligned}
$$

where $D$ is the dimension of the space, $e$ is the electron charge, $\xi(0)$ is the Ginzburg-Landau (GL) coherence length of the superconductor, $\tau_{\text {rel }}$ is a dimensionless constant which describes how long the fluctuation Cooper pairs live in comparison with the prediction of BCS theory, $k_{B}$ is the Boltzmann constant, $U$ is the voltage difference, $L$ is the length of the sample, and

$$
\epsilon=\frac{T-T_{c}}{T_{c}}, \quad g=\frac{f^{2}}{12}, \quad f=\frac{\pi}{8} \frac{e E \xi(0)}{k_{B} T_{c}} \tau_{\text {rel }}, \quad E=\frac{U}{L} .
$$

The analysis of Eq. (3) shows that below $T_{c}$ where $\epsilon<$ 0 the differential conductivity is really negative as it is necessary for the work of the suggested current oscillator. The GL theory is formally applicable only close to $T_{c}$ for $|\epsilon| \ll 1$, but qualitatively its results can be used even far from $T_{c}$. In other words, the differential conductivity will remain negative even if the accuracy of the TDGL formula Eq. (31) is not very high. We have to mention that TDGL equation is derived from BCS theory as a result of some approximations and for some cases it could be only a convenient model equation. We also wish to point out that the dimension of the current density depends on the dimension of the space $\left[j_{D}\right]=\mathrm{A} / \mathrm{m}^{D-1}$ : for a bulk sample $\left[j_{3}\right]=\mathrm{A} / \mathrm{m}^{2}$, for thin films with a thickness $d_{\text {film }} \ll \xi(\epsilon)\left[j_{2}\right]=\mathrm{A} / \mathrm{m}$, and for a wire with a crosssection $\ll \xi^{2}(\epsilon)$ the current density is just the current $\left[j_{1}\right]=$ A. Here

$$
\xi(\epsilon)=\frac{\xi(0))}{\sqrt{|\epsilon|}}
$$

is the temperature dependent coherence length. It is also convenient to introduce a temperature dependent Cooper pair life-time $\tau(\epsilon)$

$$
\tau(\epsilon)=\frac{\tau(0)}{|\epsilon|}, \quad \tau(0)=\frac{\pi}{16} \frac{\hbar}{k_{B} T_{c}} \tau_{\text {rel }},
$$

where the numerical coefficient $\pi / 16$ is a result of the microscopic BCS theory. Analogously, it is convenient to introduce a dimensionless temperature dependent electric field

$$
f_{\epsilon}=\frac{f}{|\epsilon|^{3 / 2}}=\left|e^{*} E\right| \xi(\epsilon) \tau(\epsilon) / \hbar \ll 1,
$$

where $\left|e^{*}\right|=2|e|$ is the charge of the Cooper pair. The dummy parameter of the integration in Eq. (3) has a physical meaning of a dimensionless time $u=t / \tau(0)$, and analogously, one can introduce another dimensionless time $v=t / \tau(\epsilon)$.

The present theory is applicable for every superconductor which is homogeneous enough in order to avoid nucleation of the superconducting phase. However, we consider as the most promising the cuprate high- $T_{c}$ superconductors containing as a main structural detail superconducting $\mathrm{CuO}_{2}$ planes, such as $\mathrm{YBa}_{2} \mathrm{Cu}_{3} \mathrm{O}_{7_{\delta}}$ and $\mathrm{Bi}_{2} \mathrm{Sr}_{2} \mathrm{CaCu}_{2} \mathrm{O}_{8}$ superconductors which have $T_{c} \approx 90 \mathrm{~K}$ and can be cooled by liquid nitrogen using working temperatures $T=80 \mathrm{~K}$ and reduced temperature $\epsilon \simeq-0.1$. The coherence lengths in $\mathrm{CuO}_{2}$ plane are typical for other $90 \mathrm{~K}$ cuprates $\xi_{a b}(0) \simeq 2 \mathrm{~nm}$. All high- $T_{c}$ cuprates have a significant anisotropy but $\mathrm{Bi}_{2} \mathrm{Sr}_{2} \mathrm{CaCu}_{2} \mathrm{O}_{8}$ is extremely anisotropic. Even for small reduced temperatures $|\epsilon| \simeq 0.1$ the coherence length perpendicular to the $\mathrm{CuO}_{2}$ plane can be smaller than the distance $s$ between double planes $\mathrm{CuO}_{2}$. In this case, every double planes operate approximately as an independent two dimensional (2D) layer and the number of layers $N_{1}$ depends on the film thickness $N_{\mathrm{l}}=d_{\mathrm{film}} / \mathrm{s}$. If the superconductor is a strip with a width $w$, patterned from a layered superconductor we have for the total current

$$
I=\frac{w d_{\text {film }}}{s} j_{2}(E) .
$$

Such a way for the differential conductance of the sample we obtain

$$
\sigma_{\mathrm{diff}}(U)=\frac{d i(U)}{d U}=\sigma_{N}+\frac{e^{2} \tau_{\mathrm{rel}} w d_{\mathrm{film}}}{16 \hbar s|\epsilon|} S\left(g_{\epsilon}\right),
$$

where the universal function

$$
S_{\text {diff }}\left(g_{\epsilon}\right)=\int_{0}^{\infty}\left(1-2 g_{\epsilon} v^{3}\right) \exp \left(\operatorname{sign}(-\epsilon)-g_{\epsilon} v^{3}\right) d v
$$

have to be calculated only once for the $\operatorname{sign}(-\epsilon)= \pm 1$. The negative differential conductivity arises only for supercooled below $T_{c}$ superconductors, and in this case $\operatorname{sign}(-\epsilon)=1$. In Eq. (9) the electric field is parameterized by the dimension parameter

$$
g_{\epsilon}=\frac{g}{|\epsilon|^{3}}=\frac{1}{12} f_{\epsilon}^{2}=\frac{1}{12|\epsilon|^{3}}\left(\frac{\pi e U \xi(0) \tau_{\mathrm{rel}}}{8 k_{B} T_{c} L}\right)^{2} .
$$

In order to have a significant negative differential conductivity this parameter should be small enough $g_{\epsilon} \ll 1$. This means that close to the critical region the applied DC voltage $U$ should be small enough. 
The fluctuations are stronger in low dimensional systems, that is why another realization of the negative differential conductivity in superconductors could be a nanostructured stripe of conventional superconductor on nanowires. Consequently, we have to use one dimensional (1D) formula for the current. Analogously, for layered superconductors we can use Lawrence-Doniach theory which can be interpolated by some space dimension $2 \leq D \leq 3$. It is necessary to use disordered conventional superconductors or cuprates in order to have smaller normal current.

\section{DESCRIPTION OF THE OSCILLATIONS}

In order to illustrate how electric oscillations can be generated by a supercooled superconductor we will use the simplest possible electric scheme used in generators with tunnel diodes $\stackrel{8}{-}$ For a pedagogical explanation of this scheme and the Van der Pol equation see also the excellent textbook 9

The superconductor is connected in parallel with one resistor having resistance $R$ and with one capacitor having capacity $C$. Those 3 elements are sequentially connected in a circuit with one inductance $L$ and a battery with electromotive force $\mathcal{E}$. For a static current the voltage on the superconductor, the capacitor and the resistor is just the voltage of the battery $U=\mathcal{E}$. In this static case, the voltage of the inductance is zero.

Let us now consider what will happen if the superconductor is supercooled. The whole circuit is cooled below $T_{c}$, but we consider that in the beginning superconductor is in the normal state. Imagine that it is heated by a short current or a laser impulse. We will analyze the fluctuations of the voltage of the superconductor taking into account the static solution $U(t)=\mathcal{E}+x(t)$. The deviation from the static solution $x \equiv U(t)-\mathcal{E}$ obeys the differential equation

$$
C \frac{d^{2}}{d t^{2}} x+\left[\frac{1}{R}+\sigma_{N}+\sigma_{\operatorname{diff}}(\mathcal{E}+x)\right] \frac{d}{d t} x+\frac{1}{L} x=0,
$$

cf. Ref. 9. After introducing the auxiliary variable

$$
y(t) \equiv \frac{d}{d t} x(t)
$$

the second equation of this system of ordinary differential equations reads as

$$
\frac{d}{d t} y(t)=-\nu(v) y-\omega^{2} x
$$

where

$$
\nu(x) \equiv \frac{1}{C}\left[\frac{1}{R}+\sigma_{N}+\sigma_{\mathrm{diff}}(\mathcal{E}+x)\right], \quad \omega=\frac{1}{\sqrt{L C}} .
$$

For a moderate accuracy necessary for the modelling of electronic circuits we can use some adaptive Runge-Kutta method, $\stackrel{10}{10}$ or a simple empirical formula for the time step which follows the characteristic frequencies of the circuit

$$
\Delta t=0.1 / \sqrt{\nu^{2}(x)+4 \omega^{2}} .
$$

The physical restrictions for the high frequencies are related only to applicability of the TDGL equation and the static formulae for the current response. The static response approximation used for the derivation of Eq. (3) is applicable for $\omega \tau(\epsilon) \ll 1$. This means that for a high- $T_{c}$ superconductor this generator can operate for the whole radio frequency range and even in the far infrared region

$$
\omega \ll|\epsilon| k_{B} T_{c} / \hbar \simeq 1 / \tau(\epsilon) .
$$

Let us now describe the appearance of the oscillations when the superconductor is supercooled. The total differential conductivity of the circuit becomes zero at the temperature $T_{g}$, determined by solution of the equation

$$
-\sigma_{\mathrm{diff}}\left(\mathcal{E}, T_{g}\right)=\frac{1}{R}+\sigma_{N}\left(T_{g}\right) .
$$

Further cooling leads to appearance of NDC, the static solution $x(t)=0$ looses stability, and the voltage in the circuit starts to oscillate. The main difference with the tunnel diode devices is that for superconductors we have no definite region of negative conductivity as a function of the voltage. The current continuously increases when the voltage decreases down to zero voltage. It is quite possible that amplitude of the oscillations will be limited only by ohmic heating of the sample. For thin films the heat current is determined mainly by the boundary resistance $\mathcal{R}_{h}$ of the interface of the superconductor and the insulator substrate. For high frequencies we can average the dissipated power and calculate the local increasing of the temperature of the superconductor above the ambient temperature

$$
\Delta T=\frac{\langle I(t) U(t)\rangle_{t}}{\mathcal{R}_{h}} .
$$

Such a way we obtain a self-consistent equation for the reduced temperature

$$
\epsilon=\frac{\Delta T+T-T_{c}}{T_{c}}
$$

which have to be substituted in the formula for the differential conductivity Eq. (8). The complex problem of the temperature and electric field oscillations can be easily simulated on a computer in order to optimize the parameters of the device and the initial stage of the experimental research.

One can also speculate what will happen if we start with a superconducting sample. Applied voltage will destroy the superconductivity but it is also possible that a space inhomogeneous state will appear. It is difficult to predict the behaviour of the system when having a problem related to the domain structure. That is why an experimental investigation is needed. 


\section{PERFORMANCE OF THE GENERATOR}

The most important prerequisite for the realization of NDC by supercooled superconductors is to keep the superconductor in the metastable normal state by preventing its active part from transition to the superconducting state which is thermodynamically stable below $T_{c}$. The sample has to be clean from defects, for instance pin holes, that can nucleate locally the superconductivity. Special efforts have to be applied to the contacts of the superconductor sample where the current density is low and these regions could be a source of nucleation of superconducting domains. We consider that only the central working region of the sample should be superconducting. The contact area should have inserted depairing defects. Such defects could be the magnetic impurities for conventional superconductors, $\mathrm{Zn}$ in $\mathrm{CuO}_{2}$ plane, etc. Oxygenation of $\mathrm{YBa}_{2} \mathrm{Cu}_{3} \mathrm{O}_{7-\delta}$ superconductors or changing stoihiometry are also a tool to change $T_{c}$ locally. Overdoped and underdoped cuprate thin films will have opposite behaviour with respect to the stability of appearance of space inhomogeneous domains of the superconducting and normal phases.

The amplitude of the oscillations can also be restricted by a current limiter with a maximal current $I_{c}(T)$, a narrow superconducting wire, Josephson junction, or narrow superconducting strip sequentially switched to the inductance of the resonance circuit. In a rough approximation the resistance of the limiter $R_{\mathrm{i}}=R_{0} \theta\left(I_{c}(T)-I\right)$ is switched when the current $I(t)$ becomes bigger than the critical $I_{c}(T)$. Such additional amplitude dependent dissipation will prevent the sample to pass into normal state when $U(t)=\mathcal{E}+x(T)=0$. For such a small modification of the circuit we have to solve the system of equations

$$
\begin{aligned}
\mathcal{E} & =L \frac{d I}{d t}+R_{0} \theta\left(I_{c}(T)-I(t)\right) I(t)+U(t), \\
I(t) & =\left(\frac{1}{R}+\frac{1}{R_{N}}\right) U(t)+C \frac{d U(t)}{d t}+I_{\mathrm{f}}[U(t)],
\end{aligned}
$$

where, if necessary, the Boltzmann equation can be solved in the general case in order to obtain the high frequency functional for the fluctuation current $I_{\mathrm{fl}}[U(t)]$ and eventually the self-interaction between fluctuation Cooper pairs has to be taken into account.

In the paper by Gor'kov ${ }^{6}$ were mentioned some early experiments for observation of oscillations in superconductors close to $T_{c}$. Those experiments give a hint that creation of oscillations in supercooled superconductor is also possible. But the problem requires detailed experimental investigation. Very often NDC leads to space inhomogeneities instead of time oscillations and we consider that both regimes can be realized. To avoid the appearance of space inhomogeneities one can also use narrow (nanostructured) region of supercooled superconductor which will be analogous to the tunnelling region of a tunnel diode. The length of the superconducting area should be comparable with the coherence length of the superconductor $\xi(\epsilon)$.
Fluctuation effects in conventional superconductors are very weak, with the exception of some very disordered films with a high Ohmic resistance. They cannot be effectively used in $\mathrm{THz}$ region due to their relatively smaller critical temperature $T_{c}$, the superconducting gap and the energy scale in general. However, systematic investigations of conventional superconducting nanostructures can be very important step for creation of electric oscillators with a superconductor as an active element. In experiments with gaseous plasma and semiconductors the NDC can be reached by various viable methods and it is not strange that the same could happen for superconductors. The investigation of current-voltage characteristics of superconducting nanowires ${ }^{12}$ is promising. We consider that for superconducting nanowires could be taken into account the phase slip centers, the domains of different temperature, or even some possible modification of time-dependent Ginzburg-Landau theory might be done. A detailed physical picture can be drawn only after detailed investigations. We suggest conventional superconductors to be used to demonstrate that NDC can be reached and thus to stimulate further investigation of cuprate nanostructures. Successful experiments with high- $T_{c}$ superconductors can trigger significant applications in the $\mathrm{THz}$ range as mentioned above. When the current oscillator is realized by a cuprate the resonance circuit (the capacitor, the inductive coil and the current limiter) can be patterned in a single chip from the same submicron cuprate layer. We can generate electric oscillations and $\mathrm{NDC}$ to be well inside the $\mathrm{THz}$ region only by nanostructured cuprates because the maximal frequency at which NDC can exist is proportional to the maximal value of the superconducting gap and critical temperature $T_{c}$.

\section{POSSIBLE APPLICATIONS}

The described principle of generation of current oscillations can be realized in superconducting electronics. In general, the negative differential conductivity (NDC) could be a useful tool in the electronics using superconductors as an active element.

We consider that the generation of submillimeter electromagnetic waves by high- $T_{c}$ superconductors is quite possible. Thin superconducting layers can be electrostatically doped and in this context some preliminary research on superconducting field effect transistors can be extremely helpful for modulation of the oscillations. There are good prospectives for application in the wireless communications. We consider also that in the regime of supercooling some old samples could become working transistors.

Fluctuations are more important for the low dimensional systems. The fact that NDC can be observed in conventional nanostructured superconductors is promising. In this case the $1 \mathrm{D}$ theory can be directly used or one can easily perform summation on the perpendicu- 
lar modes of the Cooper pair wave guide. Shortly said, the combination of nanostrip, capacitor and inductance patterned into one chip or the plasma modes of the superconducting stripe, could be considered as the smallest current oscillator performed for demonstration purposes.

The device constructed upon our instructions will be very sensitive to the temperature and might be stabilized to oscillate near the critical temperature $T_{c}$. It should not only prove our theory but could be used as well as a superconducting bolometer. The sensitivity to temperature variations around $T_{c}$ opens opportunities for optoelectronic applications.

The negative differential conductivity can be investigated in the case of zero temperatures $T \ll T_{c}$ and the critical behaviour in small electric field is appropriate for investigation of the quantum criticality. This is an evidence that development of the applied research can in return stimulate further development of the initializing theory.

The described idea can be realized not only for the inplane conductivity of $\mathrm{MgB}_{2}$ and $\mathrm{CuO}_{2}$ containing high$T_{c}$ superconductors but also for the currents in the so called c-direction perpendicular to the $\mathrm{CuO}_{2}$ layers. For $\mathrm{Bi}_{2} \mathrm{Sr}_{2} \mathrm{CaCu}_{2} \mathrm{O}_{8}$ the NDC can be coupled with plasma resonances having frequencies lower than the superconducting gap. This coupling leads to natural realization of voltage induced FIR oscillations with low dissipation.

The nonlinear conductivity in supercooled regime can be used also for frequency mixers and transistors. The possibility to operate in terahertz range (loosely defined by the frequency range of 0.1 to $10 \mathrm{THz}$ ) using hight- $T_{c}$ superconductors looks very promising. Recently Ferguson and Zhang ${ }^{11}$ mentioned that the lack of high-power, low-cost, portable room-temperature $\mathrm{THz}$ source is the most significant limitation of the modern $\mathrm{THz}$ systems. They consider that the narrow band $\mathrm{THz}$ sources are crucial for high-resolution spectroscopy applications. In addition, the authors are stressing that this kind of sources have broad potential applications in telecommunications and are particularly attractive for extremely high bandwidth intersatellite links. Our invention is designed to fill this gap with its simple theoretical explanation and easy for performance practical proposal.

The $\mathrm{THz}$ region of the electromagnetic waves (the frequencies between $100 \mathrm{GHz}$ and $10 \mathrm{THz}$ or wavelengths between $3 \mathrm{~mm}$ and $30 \mu \mathrm{m}$ lies between radiofrequencies and optics and up to now is not so well developed for applications and research as neighbouring frequency ranges. The state of the art review on the application and sources of $\mathrm{THz}$ radiation and the need of improvement and new sources is given in a recent review article by Mueller 13 The author points out that practical application of $\mathrm{THz}$ radiation is in initial stage and only some reliable sources are available. That is why every new principle of $\mathrm{THz}$ wave generation can be not only significant theoretical input but will have important technical applications in the near future. In the review $\underline{13}$ is mentioned also that recently the investigators are pursuing potential $\mathrm{THz}-$ wavelength applications in many fields: quality control; biomedical imaging; THz tomographic imaging in mammography; passengers' screening for explosives at the airports; detecting the presence of cancerous cells; complex dynamics involved in condensed matter physics; molecular recognition and protein folding; environmental monitoring; plasma diagnostics; Antarctic submillimeter telescope which will be used to measure interstellar singly ionized nitrogen and carbon monoxide during the polar winter; significant part of the photons emitted since the Big Bang fall also in $\mathrm{THz}$ region - continuous-wave $\mathrm{THz}$ sources can be used to help study these photons; $\mathrm{THz}$ imaging using time domain spectroscopy developed in Lucent Technologies' Bell Laboratories - it uses the greatly varying absorption characteristics from material to material; NASA's AURA satellite measuring the concentration and distribution of hydroxyl radical (OH-) in the stratosphere, a crucial component in the ozone cycle, etc.

At the end, we would like to draw reader's attention to another possible application. In a semiconductor nanostructure with two dimensional electron gas and an appropriate grating coupler $\mathrm{THz}$ electromagnetic waves can be transmitted in hyper sound phonons - such a way we will have an ersatz phonon laser useful for phonon spectroscopy as well. 14

In conclusion we consider that investigation of voltage biased conductivity of nanostructured superconductors is very perspective theme of the fundamental science promising viable variety of technical applications.

\section{ACKNOWLEDGMENTS}

We would like to especially thank V. Mishonova who has initiated this challenging project and contributed to its completion. Special thanks go to W. Bird and I. Roelants for their cooperation and stimulating discussions. One of the authors (TMM) is thankful to D. Cocke for pointing out the Ref. 13 and to T. Donchev for the critical reading of the manuscript.
* E-mail: Mishonov@phys.uni-sofia.bg

1 A. Larkin and A. Varlamov, "Fluctuation Phenomena in Superconductors", cond-mat/0109177 in Physics of Conventional and Unconventional Superconductors, Edited by K. Benneman and J. B. Ketterson (Springer, Berlin, 2002).
2 T. Mishonov and E. Penev, "Thermodynamics of Gaussian fluctuations and paraconductivity in layered superconductors", cond-mat/0004023 Int. J. Mod. Phys. 14, 3831 (2000).

3 T. Mishonov and D. Damianov, Czech. J. Phys. 46 (Suppl. 
S2) 631 (1996); D. Damianov and T. Mishonov, Superlattices and Microstructures 21, 467. (1997).

4 T.M. Mishonov, A.I. Posazhennikova and J.O. Indekeu, "Fluctuation conductivity in superconductors in strong electric fields", cond-mat/0106168 Phys. Rev. B 65, 064519 (2002).

5 T.M. Mishonov, G.V. Pachov, I.N. Genchev, L.A. Atanasova, and D.C. Damianov, "Kinetics and Boltzmann kinetic equation for fluctuation Cooper pairs", cond-mat/0302046 Phys. Rev. B 68, 054525 (2003); T.M. Mishonov and M.T. Mishonov (03.03.2003), "Generation of Electric Oscillations by Continuous, Supercooled Superconductrors with an Applied Voltage", W02004079893.

${ }^{6}$ L.P. Gor'kov, Zh. Eksp. Theor. Phys. Pis. Red. 11, 52 (1970) [Sov. Phys.-JETP Lett. 1132 (1970)] Eq. (5).

7 J.R. Tucker and B.I. Halperin, Phys. Rev. B 3, 3768 (1971).

8 W.F. Chow, Principles of Tunnel Diode Circuits (Wiley, New York, 1964).
9 R.H. Enns and G.C. McGuire, Computer Algebra Recipes. A Gourmet's Guide to the Mathematical Models of Science, (Springer, Berlin, 2001) Sec. 7.4.1, p. 445, Fig. 7.22, "The Bizarre World of the Tunnel Diode Oscillator."

${ }^{10}$ W.H. Press, S.A. Teukolsky, W.T. Vetterling, and B.P. Flannery, Numerical Recipes in Fortran 7r: the Art of Scientific Computing, Second Edition, Volume 1 of Fortran Numerical Recipes, (Cambridge University Presss, 2001), p. 704, Sec. 16.1, "Runge-Kutta Method."

11 B. Ferguson and X.-C. Zhang, Review article: "Materials for teraherz science and technology", Nature Materials, 1, 26 (2002); e-mail: zhangxc@rpi.edu

12 S. Michotte, S. Mátéfi-Tempfli and L. Perrot, Supercond. Sci. Technol. 16, 557 (2003).

13 E. Mueller, "Terahertz Radiation: Applications and Sources", The Industrial Physicist (August/September 2003) pp. 27-29; e-mail: eric.mueller@coherentinc.com

14 T.M. Mishonov, "Acoustic-phonon emission by twodimensional plasmons", Phys. Rev. B 43, 7787 (1991). 